\title{
Electromagnetic stimulation in psychiatry
}

\author{
Klaus P. Ebmeier \& Julia M. Lappin
}

One hundred years ago, D'Arsonval and Beer first described the effects of magnetic fields on human brain function. Placing one's head into a powerful magnet produced phosphenes, vertigo or even syncopes (George \& Belmaker, 2000). However, only since 1985 has the technology of fast discharging capacitors developed sufficiently to generate reproducible effects across the intact skull, with peak magnetic field strengths of about 1-2 tesla (Barker et al, 1985). The headline-grabbing news has been about therapeutic applications of transcranial magnetic stimulation (TMS), but in the meantime a revolution in functional brain research has taken place, based on the manipulation of brain activity by focused magnetic fields. TMS applied in this way is, in a manner of speaking, brain imaging in the reverse. While common modes of functional brain imaging, such as positron emission tomography (PET) and functional magnetic resonance imaging (fMRI), demonstrate associations between brain metabolic activity and 'brain tasks', the causal interpretation of such associations can be difficult. Is the frontal lobe activation observed during a memory task, for example, necessary for performing the task, or does it correspond to monitoring activity that runs parallel to task performance proper? If, on the other hand, focal brain activation during TMS results in a muscle twitch, there is no doubt that stimulation of at least some of the neurons within the magnetic field is sufficient cause for the observed movement. Functional neuroimaging is now often combined with TMS, carried out in the same session in order to exploit the complementary strengths of the methods. Although direct stimulation of association (as opposed to motor or sensory) cortex does not usually result in an observable response, TMS applied in repetitive trains can produce reversible 'lesions'. By interfering with tasks that are dependent on the functioning of the stimulated neurons, it can thus contribute to the localisation of brain function.

In this article we will give the reader a concise overview, not only of the as yet immature efforts to treat psychiatric conditions with TMS, but also of the use of TMS as a research tool to clarify the brain mechanisms of psychiatric illness (see also Lisanby et al, 2000).

\section{Physics and physiology of TMS}

Barker et al (1985) demonstrated that a coil with a strong electric current placed over the vertex of a normal subject could generate a motor response in certain hand muscles. This effect is based on electromagnetic induction: a change in electric current generates a magnetic field, which in turn induces a current in a second conductor brought into the magnetic field. Magnetic fields penetrate body tissue without the loss and dissipation of energy associated with electrical stimulation. Apart from stimulation of superficial muscles and nerves, there is no generation of heat or pain as in electrical stimulation. The stimulus delivered to the cortex mainly depends on distance, that is, the thickness of the skull and other tissue layers, rather than factors such as conductance. The strength of the magnetic field decays, so that typically only the top $2 \mathrm{~cm}$ of the cortical cell layer are likely to be stimulated. Theoretically, deeper layers could also be stimulated if the field strength was increased,

Klaus P. Ebmeier is Professor of Psychiatry at the University of Edinburgh (Department of Psychiatry, Kennedy Tower, Morningside Park, Edinburgh EH10 5HF0). His research interests include the general psychiatry of adults and old age and neuroimaging. Julia M. Lappin is a senior house officer in psychiatry in Edinburgh. Her research interests are in neuroscience. 
but this would result in overstimulation of superficial layers, resulting in seizure induction (see below).

Typically, the current running through the insulated coil is of the order of 10000 amperes, with a magnetic field strength of 1 tesla, a specific absorption per pulse of $2.46 \mathrm{~mJ} / \mathrm{kg}$ and a specific absorption rate at $1 \mathrm{~Hz}$ stimulation frequency of 2.46 $\mathrm{mW} / \mathrm{kg}$ brain tissue, as described by Bohning (2000). The simplest coils are circular with a magnetic field the shape of a torus (ring doughnut) enveloping the coil. Unless the coil is held on end, the magnetic field will extend over areas of cortex (e.g. 5-7 cm diameter) that are larger than desirable. A practicable compromise is the figure-of-eightshaped coil that has a magnetic field of two superimposed tori, with a conical shape above and below the crossover of the two circles that has an effective diameter of a couple of centimetres.

The neuronal mechanisms of TMS are not entirely clear. Changing electric fields are generated parallel and possibly also perpendicular to the axon, which may cause neuronal discharges. In reality, within a given volume of stimulation, axons will be running in different orientations, maybe even bending along their course, so that the net effect of TMS over a particular cortical area is difficult to predict. The effects of TMS on short fibres, cell bodies or dendrites are not known (Bohning, 2000).

A central concept in practical TMS is the motor threshold, a measure that varies between subjects owing to skull thickness and head shape, but also to functional factors such as cortical excitability, medication and acute brain state, for example, induced by recent exercise. It allows for an intrinsic calibration of stimulus strength, which is crucial, both for safety reasons (see below) and in order to use comparable stimulus strengths in experiments conducted between subjects. By convention the motor threshold is defined by the stimulus strength that evokes five out of 10 muscle potentials, usually in a hand muscle, with an amplitude of $50 \mu \mathrm{V}$ or more. The mapping of the position with the best electromyogram (EMG) response for a given muscle, such as the abductor pollicis brevis, and the determination of the threshold stimulus strength require some skill and experience in neurophysiology, which makes TMS not as easily accessible to the clinician as, for example, electroconvulsive therapy (ECT).

A number of measures derived from stimulation of the motor cortex have been used to derive diagnostic markers. Cortical excitability, examined with TMS, can be defined as an intracortical mechanism that is observable through a variable motor response to standardised TMS. The most obvious measure of excitability is the EMG amplitude after a stimulus above motor threshold. Responses to standard stimuli can differ because of the varying contributions of excitatory and inhibitory input from interneurons to the primary motor cortex. Because TMS mainly acts via inter-neurons, it is an ideal method to assess cortical excitability. The TMS response is dependent on pre-stimulus muscle activity, in that the amplitude of the motor evoked potentials (MEP) is enhanced after exercise and reduced during fatigue. Previous magnetic stimulation also affects subsequent TMS response: a short interval (10-40 ms) between a first and a second stimulus over the same point is associated with response enhancement; longer intervals (40-200 ms) are associated with a suppression of the second response. It is presently believed that the mechanisms responsible for these phenomena are cortical in origin (Ziemann \& Hallet, 2000). A similar, although not identical, phenomenon is the so-called silent period, a period of EMG silence after a TMS stimulus. Similar to single stimuli, repetitive TMS (rTMS) has effects of its own on cortical excitability. Many investigators now assume that at low frequencies $(<1 \mathrm{~Hz})$ there is a reduction in the brain activity of the underlying areas (quenching; Chen et al, 1997), whereas at higher frequencies $(>5 \mathrm{~Hz})$ excitability increases, in extreme cases to the point of facilitating excitation of adjacent brain areas or even grand mal seizures (Pascual-Leone et al, 1993).

\section{Safety issues}

The most serious safety concern during TMS is the possibility of seizure induction by stimulation, in particular repetitive stimulation at higher frequencies $(>5 \mathrm{~Hz})$. There have been a small number of reported cases where repetitive TMS has resulted in self-limiting grand mal seizures. Factors associated with higher seizure risk include: evidence of brain pathology, for example, previous stroke or tumour; certain medication that lowers the seizure threshold (e.g. antipsychotic or antidepressant drugs); untreated epilepsy; first-degree relative with idiopathic epilepsy; stimulation characteristics, such as high stimulation strength and frequency; long stimulus trains; and short inter-train intervals. Although the immediate medical risk associated with a seizure may be manageable, the occurrence of seizures in a person without the diagnosis of epilepsy may have serious repercussions, for example, for his or her ability to drive and for certain insurances. As with all magnetic fields, special precautions have to be taken to exclude patients with pacemakers, metallic implants and other contraindications to entering magnetic fields. Because little 


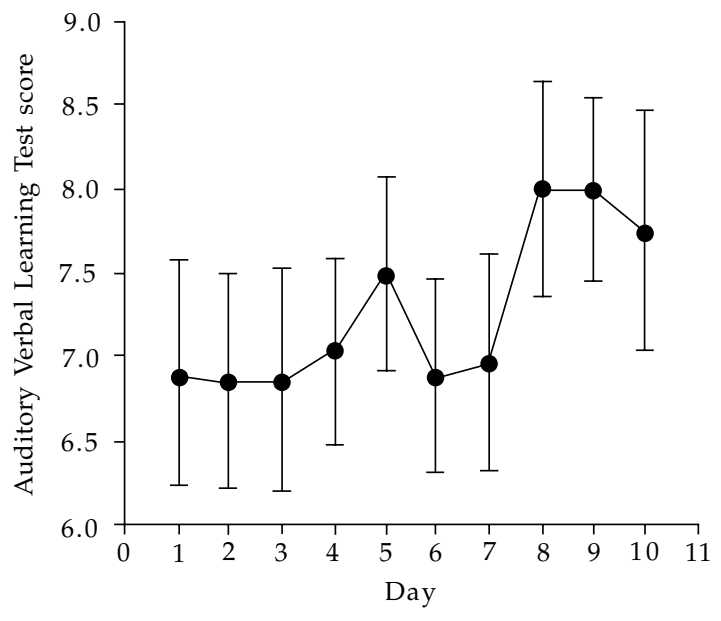

Fig. 1 Verbal memory performance during a 10-day course of rTMS in depressed patients

is known about possible teratogenic effects most investigators exclude pregnant patients (and indeed TMS operators). It has been argued, however, that TMS may be a safer treatment, particularly in pregnancy, than ECT or medication (Nahas et al, 1999).

Stimulation over an active portion of cortex can disrupt the function of this structure (Pascual-Leone \& Hallett, 1994). The obvious question therefore is whether rTMS in the course of, for example, antidepressant treatment, has cognitive side-effects. Lorbeerbaum \& Wassermann (2000) report that studies have generally been negative, or have found changes only within hours of treatment. They conclude that the chance of producing excitotoxicity with rTMS is very remote. During antidepressant treatment any possible deleterious effects of rTMS will be confounded by practice effects and the effect of recovery from depression over the course of treatment (see Fig. 1).

The click associated with coil discharge can affect hearing for hours after the treatment, so most investigators give their subjects earplugs (coil placement is often in close proximity to the ears). The most common side-effect, however, is headache, presumably owing to local superficial stimulation of muscles and nerves under the coil. Such headaches respond to standard analgesia and, if necessary, to a reduction of stimulation strength.

\section{Investigative use in schizophrenia}

The syndromes of schizophrenia have obvious motor components. Cortical inhibitory processes may be abnormal in this patient group, and in treatment-naïve patients with schizophrenia there appears to be a shortening of latency in compound MEP (cMEP) triggered by TMS over the motor strip (Puri et al, 1996). Although medication may clearly play a role in motor changes in schizophrenia, cMEP latency does not seem to be affected (Davey et al, 1997). However, the silent period after TMS was affected by medication, in that the latency of maximal suppression of the voluntary EMG silent period was increased (Davey et al, 1997). This is in contrast to previous reports in (drug-induced) parkinsonism where the silent period is shortened, so that sampling issues or methodological differences may account for this finding. There have been suggestions that callosal function is abnormal in schizophrenia. Boroojerdi et al (1999) used TMS to examine 10 patients with schizophrenia on medication and found that ipsilateral stimulation at 1 stimulus/5 s over the hand area induced EMG suppression in the voluntarily activated muscle. This inhibition was delayed in patients matched for age and gender (but probably not IQ) with healthy volunteers. The duration of inhibition was also significantly prolonged in patients. The significance of these studies is difficult to assess at the moment. Small, biased samples and the presence of medication may have caused spurious results, so that a replication of the experiments is necessary.

\section{Investigative use in affective disorders}

Brain metabolism is usually reduced in depression. Any associated reductions in cortical excitability can be examined with TMS. Samii et al (1996) examined patients with depression who did not show the typical enhancement of EMG response after exercise, and this finding was replicated by Shajahan et al (1999a). As Samii et al found similar results in patients with chronic fatigue syndrome, the specificity of the finding is in question, but Shajahan et al (1999b) were able to show that the changes observed in depression are reversible with recovery from the symptoms. This is consistent with the observation of reversible weakness and retardation in depression. Steele et al (2000) found that patients with depression had a significantly longer silent period after TMS than matched controls, suggesting an increased inhibitory tone in the motor cortex. The use of TMS in depression research promises to provide objective measures of motor impairment independently of possible motivational factors, which, it may be argued, confound behavioural experiments. 


\section{Therapeutic use in schizophrenia}

Early therapeutic studies of TMS in schizophrenia have been summarised by Nahas et al (2000. Geller et al (1997) reported transient improvement in two patients in an open trial of 10 patients with schizophrenia. Nahas et al also found transient decreases in the Scale for Assessment of Negative Symptoms (SANS) and Continuous Performance Task (CPT) scores in seven subjects with schizophrenia with negative symptoms after $20 \mathrm{~Hz}$ (presumably excitatory) stimulation over the left dorsolateral prefrontal cortex. Klein et al (1999a) treated 35 patients with schizophrenia with right prefrontal rTMS at $1 \mathrm{~Hz}$ or sham treatment. No significant difference in outcome was found between the two groups. Most recently, Hoffman et al (2000) entered 12 patients with schizophrenia and auditory hallucinations into a crossover trial of $1 \mathrm{~Hz}$ TMS. Stimulation was over the left temporo-parietal cortex, half way between T3 and P3 in the 10-20 electrode placement system. This area was chosen as the presumed site of cortical overactivity during auditory hallucinations. All patients had daily auditory hallucinations without remission for at least 6 months before entering the study, either continuously (three) or intermittently (nine). The patients were randomised into an active treatment and a sham treatment arm (coil angled away from the head by $45^{\circ}$ ) and crossed over into the complementary arm after 1 week. While no effect of order of stimulation was seen, there was a significant and specific effect of active treatment on severity of hallucinations. Patients on anticonvulsants $(n=5)$ did less well. Improvement lasted between 1 day and 2 months (median 4.5 days). The small size of this study, as well as the absence of active control conditions, such as stimulation over other brain areas, make this study very preliminary. However, small studies such as this, with rational hypotheses based on available imaging evidence about abnormal brain activity during symptoms, are most likely to help our understanding of schizophrenia and may produce active treatment modalities.

\section{Therapeutic use in affective disorders}

Thus far, most therapeutic research into rTMS has studied its possible antidepressant effects. Lisanby
\& Sackeim (2000) give a good recent review. Compared with the evidence necessary for the licensing of antidepressant drugs, the number of trials, as well as the number of subjects included in them, is small and their quality is low. Although the trials are mostly underpowered $(n=6-70)$, there is no meta-analysis of results. This is mainly because the stimulation parameters employed in different studies are too diverse, leading to the familiar 'apples and oranges' problem. For example, George et al (1997) treated 12 out-patients in a blind crossover study, using $20 \mathrm{~Hz}$ over the left dorsolateral prefrontal cortex and sham treatment. The simple rationale for this approach was that neuroimaging studies had shown reduced metabolism in this brain area, and stimulation at higher frequencies was expected to increase cortical activity under the coil. Based on a rather similar hypothesis, Klein et al (1999b) treated 71 patients with depression with the slow stimulation frequency of $1 \mathrm{~Hz}$ over the right dorsolateral prefrontal cortex. Their assumption was that TMS at $1 \mathrm{~Hz}$ would reduce cortical activity in the right prefrontal cortex and bring the relative imbalance between left and right hemisphere deactivation back into equilibrium. It is clearly inadvisable to lump these two studies

Box 1 Imaging

Transcranial magnetic stimulation (TMS) functional imaging studies can be used to establish an association between activity in a neuronal network and behaviour. By transiently blocking function in a cortical structure, a causal link can be demonstrated between behaviour and regional brain function. There are various methods by which the brain can be imaged during TMS, including single photon emission computerised tomography (SPECT), positron emission tomography (PET) and functional magnetic resonance imaging (fMRI).

Measuring brain activity during TMS holds promise for the investigation of cortical connectivity and excitability in both the healthy and the disordered human brain. Changes in these parameters in relation to motor and sensory learning, cortical reorganisation following injury, possible abnormalities of connectivity in patients with schizophrenia and normal development of connections in childhood and adolescence are likely (Paus, 1999). 
Table 1 Treatment studies in depression

\begin{tabular}{|c|c|c|c|}
\hline Study & Subjects & Parameters & Outcome \\
\hline \multicolumn{4}{|l|}{ Open trials } \\
\hline Conca et al, 1996 & $\begin{array}{l}12 \text { medicated patients } \\
\text { with depression received } \\
\text { TMS }\end{array}$ & $\begin{array}{l}\text { Single-pulse } \\
\text { stimulation }(0.17 \mathrm{~Hz}) \\
\text { over multiple sites }\end{array}$ & $\begin{array}{l}\text { TMS appeared to increase the speed of } \\
\text { treatment response }\end{array}$ \\
\hline Figiel et al, 1998 & $\begin{array}{l}56 \text { patients, most } \\
\text { medication resistant }\end{array}$ & $\begin{array}{l}\text { LDLPF stimulation } \\
\text { at } 10 \mathrm{~Hz}\end{array}$ & $\begin{array}{l}42 \% \text { overall responded, with a lower } \\
\text { response rate in the elderly }(23 \%)\end{array}$ \\
\hline $\begin{array}{l}\text { Grunhaus et al, } \\
1998\end{array}$ & $\begin{array}{l}16 \text { patients received rTMS, } \\
18 \text { patients randomised } \\
\text { into standard ECT }\end{array}$ & $\begin{array}{l}\text { rTMS at } 10 \mathrm{~Hz} \text { over } \\
\text { LDLPF cortex }\end{array}$ & $\begin{array}{l}7 / 16 \text { responded to TMS, } 12 / 18 \text { to } E C T \text {; } \\
\text { ratios for psychosis patients were } 2 / 8 \\
\text { and } 7 / 8 \text {; for non-psychosis patients, } \\
5 / 8 \text { and } 5 / 10\end{array}$ \\
\hline \multicolumn{4}{|c|}{ Blind controlled trials } \\
\hline $\begin{array}{l}\text { Pascual-Leone } \\
\text { et al, } 1996\end{array}$ & $\begin{array}{l}17 \text { treatment-resistant } \\
\text { patients with depression } \\
\text { and psychosis }\end{array}$ & $\begin{array}{l}\text { RTMS at } 10 \mathrm{~Hz} \text { over } \\
\text { various regions }\end{array}$ & $\begin{array}{l}\text { Response in } 11 / 17 \text { after LDLPF } \\
\text { treatment }\end{array}$ \\
\hline Nahas et al, 1998 & 30 patients & $\begin{array}{l}5 \mathrm{~Hz}, 20 \mathrm{~Hz} \text { or sham } \\
\text { TMS over LDLPF } \\
\text { cortex }\end{array}$ & $\begin{array}{l}\text { Active treatments showed a moderate } \\
\text { effect compared with placebo, but there } \\
\text { was no difference between frequencies }\end{array}$ \\
\hline $\begin{array}{l}\text { Padberg et al, } \\
1999\end{array}$ & $\begin{array}{l}18 \text { treatment-resistant } \\
\text { patients }\end{array}$ & $\begin{array}{l}\text { Compared } 0.3 \mathrm{~Hz} \text { with } \\
10 \mathrm{~Hz} \text { TMS over } \\
\text { LDLPF cortex and } \\
\text { sham TMS }\end{array}$ & $\begin{array}{l}\text { Modest but significant improvement in } \\
\text { the single-pulse group compared with } \\
\text { placebo, but no difference between the } \\
\text { active treatments }\end{array}$ \\
\hline Klein et al, $1999 b$ & 71 patients & $\begin{array}{l}1 \mathrm{~Hz} \text { over right } \\
\text { prefrontal cortex }\end{array}$ & $\begin{array}{l}\text { Response rate of } 17 / 36 \text { for the active and } \\
6 / 35 \text { for the sham treatment groups }\end{array}$ \\
\hline Loo et al, 1999 & 18 patients & $\begin{array}{l}10 \mathrm{~Hz} \text { over } \\
\text { LDLPF cortex }\end{array}$ & $\begin{array}{l}\text { Placebo response rate ( } 26 \% \text { drop in HRSD } \\
\text { scores) was as large as the improvement } \\
\text { in the active treatment group }\end{array}$ \\
\hline $\begin{array}{l}\text { Berman et al, } \\
2000\end{array}$ & $\begin{array}{l}20 \text { treatment-resistant } \\
\text { patients with depression }\end{array}$ & $\begin{array}{l}10 \text { with } 20 \mathrm{~Hz} \text { rTMS } \\
\text { over LDLPF cortex, } \\
10 \text { with sham TMS }\end{array}$ & $\begin{array}{l}\text { One patient showed a robust response } \\
\text { in the active group, none in the sham } \\
\text { treatment group }\end{array}$ \\
\hline
\end{tabular}

ECT, elecroconvulsive therapy; HRSD, Hamilton Rating Score for Depression; LDLPF, left dorsolateral prefrontal; TMS, transcranial magnetic stimulation; rTMS, repetitive TMS;

together to compute an average effect size, although they are based on similar theoretical premises.

Spontaneous remission or placebo response rates in depressive illness vary considerably (20-60\%), so that even sizeable improvement rates in open TMS studies of depression may not indicate superiority to placebo. A serious problem in evaluating TMS is the absence of a true placebo condition. Previous strategies include angling the stimulation coil away from the head surface. However, there is some doubt whether subjects cannot differentiate between flat and angled coil positions by the strength of superficial nerve and muscle stimulation. Some authors have also cast doubt on the assumption that with an angled coil no activation of cortical tissue occurs, arguing that therapeutic effects may be expected from this supposed placebo treatment (Lisanby \& Sackeim, 2000). An alternative approach involves the use of specially prepared coils that have the same amount of energy running in separate conductors in opposite directions, thereby neutralising the magnetic field. Such coils are presently being evaluated. Their advantage is that they can be placed identically to the active coil and that they produce the same stimulus-related click, but because of the absence of a net magnetic field, the surface stimulation will be different. The other important drawback of existant TMS treatment studies in depression is that none has examined long-term effects in a systematic manner. Depression is clearly often a recurring and relapsing condition, so that treatment efficacy has to be evaluated over longer periods. As TMS is generally not expected to be effective for more than a few days after discontinuation (Pascual-Leone et al, 1996), the replicability of any treatment success needs to be examined. 
With these caveats in mind, it is of interest to look at the effect sizes of the published trials that involved more than 15 patients with depression (see Table 1). Among the open trials are those of Conca et al (1996), Figiel et al (1998) and Grunhaus et al (1998). Conca et al (1996) used single pulse stimulation $(0.17 \mathrm{~Hz})$ over multiple sites in a randomised subgroup of six out of 12 patients with depression on medication. TMS appeared to increase the speed of response. Figiel et al (1998) found that $42 \%$ of 56 patients who were mostly medication-resistant responded to left dorsolateral prefrontal stimulation at $10 \mathrm{~Hz}$, with a lower response rate in the elderly subgroup of patients. Grunhaus et al (1998) compared 16 patients receiving rTMS at $10 \mathrm{~Hz}$ over the left dorsolateral prefrontal cortex with 18 patients randomised into standard ECT treatment. While he found that for patients without psychoses the response rates were comparable, ECT had the clear edge in the subgroup with psychoses. This study is clearly too small to draw any definite conclusions, but larger trials are underway.

Among the blind controlled trials, the larger ones are those of Pascual-Leone et al (1996) $(n=17)$, Nahas et al (1998) $(n=30)$, Padberg et al (1999) $(n=18)$, Klein et al $(1999 b)(n=71)$ and Loo et al $(1999)(n=18)$. The Pascual-Leone et al (1996) study will be difficult to replicate, not only because of its large effect size (response in 11/17 treatment-resistant patients with psychosis and depression), but also because of its length (5 months) and the fact that patients stayed treatment-free (apart from TMS) during the whole period. Nahas et al (1998) reported on the treatment of 30 patients with $5 \mathrm{~Hz}, 20 \mathrm{~Hz}$ or sham TMS over the left dorsolateral prefrontal cortex. The active treatments showed a moderate effect compared with placebo, but there was no difference between frequencies. Padberg et al (1999) compared single pulse TMS $(0.3 \mathrm{~Hz})$ with $10 \mathrm{~Hz}$ TMS over the left dorsolateral prefrontal cortex and placebo in 18 treatment-resistant patients. There was a modest but significant improvement in the single pulse group compared with placebo, but no difference between the active treatments. Klein et al (1999b) treated with $1 \mathrm{~Hz}$ over the right prefrontal cortex (see above) and found a response rate of $17 / 36$ for the active and 6/ 35 for the sham treatment groups. Loo et al (1999) published the only negative study, using $10 \mathrm{~Hz}$ over the left dorsolateral prefrontal cortex. Their placebo response rate $(26 \%$ drop in scores on the Hamilton Rating Scale for Depression (HRSD; Hamilton, 1960)) was as large as the improvement in the active treatment group, demonstrating the difficulty of conducting treatment trials in depression. Most recently, Berman et al (2000) reported on the treatment of 20 treatment-resistant patients with depression, 10 with active $20 \mathrm{~Hz}$ rTMS over the left dorsolateral prefrontal cortex, 10 with sham treatment. Only one patient showed a robust response in the treatment group; a further three patients showed partial responses, with reductions in HRSD score of $<45 \%$. No improvement was seen in the sham treatment group.

In summary, these are interesting preliminary results suggesting that TMS may have antidepressant effects. However, there is no convincing evidence so far that a particular stimulation mode, be it frequency or placement over the head, is superior to others. This casts doubt on any hypotheses about the action of TMS and raises the suspicion that non-specific effects may be important.

\section{Other electromagnetic modalities}

The potential hazard of seizure induction during rTMS has recently been turned into a potential strength, by using a varying magnetic field to induce seizures during magnetoconvulsive therapy (MCT) (personal communication, T. Schlaepfer, 2000). Without the vagaries of poor and variable electrical conductivity, which allows only a small proportion of the current applied during ECT to pass through the brain, MCT can focus and dose the brain stimulation more accurately and reliably, with the potential benefits of limiting stimulation to the brain structures essential for treatment response and, hopefully, reducing side-effects such as memory impairment.

Most recently, vagus stimulation by implanted pacemaker, a treatment method previously used for the control of epileptic seizures, has been applied to the treatment of depression (George et al, 2000; Rush et al, 2000). Thirty treatment-resistant patients with depression but not psychosis received an implant of a pacemaker stimulating the left cervical vagus nerve using bipolar electrodes, attached below the cardiac branch. Stimulation was mostly with $0.5 \mathrm{~ms}$ pulse-width, at $20-30 \mathrm{~Hz}$, with $30 \mathrm{~s}$ stimulation periods alternating with $300 \mathrm{~s}$ breaks. This open protocol was sustained over 10 weeks, with a response rate of $40 \%$ at end-point. Patients had failed to respond to at least two robust treatment attempts, and had an average duration of illness episode of 10 years (0.3-49.5 years). The most common stimulation-related adverse event was voice alteration, usually hoarseness, in $40 \%$; pain, coughing and dysphagia each affected $10 \%$. Considering the severity of illness, this is an encouraging result that warrants further controlled studies.

Although the treatments described above are still experimental, it is likely that ECT will be joined by other 'physical' treatments of depression in the not too distant future. 


\section{References}

Barker, A. T., Jalinous, R. \& Freeston, I. L. (1985) Non-invasive magnetic stimulation of human motor cortex. Lancet, $i$, 1106-1107.

Berman, R. M., Narasimhan, M., Sanacora, G., et al (2000) A randomized clinical trial of repetitive transcranial magnetic stimulation in the treatment of major depression. Biological Psychiatry, 47, 332-337.

Bohning, D. E. (2000) Introduction and overview of TMS physics. In Transcranial Magnetic Stimulation in Neuropsychiatry (eds M. S. George \& R. H. Belmaker), pp. 13-44. Washington, DC: American Psychiatric Press.

Boroojerdi, B., Töpper, R., Foltys, H., et al (1999) Transcallosal inhibition and motor conduction studies in patients with schizophrenia using transcranial magnetic stimulation. British Journal of Psychiatry, 175, 375-379.

Chen, R., Classen, J., Gerloff, C., et al (1997) Depression of motor cortex excitability by low-frequency transcranial magnetic stimulation. Neurology, 48, 1398-1403.

Conca, A., St. Koppi, A., König, P., et al (1996) Transcranial magnetic stimulation: a novel antidepressive strategy? Neuropsychobiology, 34, 204-207.

Davey, N. J., Puri, B. K., Lewis, H. S., et al (1997) Effects of antipsychotic medication on electromyographic responses to transcranial magnetic stimulation of the motor cortex in schizophrenia. Journal of Neurology, Neurosurgery and Psychiatry, 63, 468-473.

Figiel, G. S., Epstein, C., McDonald, W. M., et al (1998) The use of rapid-rate transcranial magnetic stimulation (rTMS) in refractory depressed patients. Journal Neuropsychiatry and Clinical Neurosciences, 10, 20-25.

Geller, V., Grisaru, N., Abarbanel, J. M., et al (1997) Slow magnetic stimulation of prefrontal cortex in depression and schizophrenia. Progress in Neuropsychopharmacology and Biological Psychiatry, 21, 105-110.

George, M. S. \& Belmaker, R. H. (2000) Historical overview. In Transcranial Magnetic Stimulation in Neuropsychiatry (eds M. S. George \& R. H. Belmaker), pp. 1-12. Washington, DC: American Psychiatric Press.

-, Wassermann, E. M., Kimbrell, T. A., et al (1997) Mood improvement following daily left prefrontal repetitive transcranial magnetic stimulation in patients with depression: a placebo-controlled crossover trial. American Journal of Psychiatry, 154, 1752-1756.

-, Sackeim, H. A., Rush, A. J., et al (2000) Vagus nerve stimulation: a new tool for brain research and therapy. Biological Psychiatry, 47, 287-295.

Grunhaus, L., Dannon, P. \& Schreiber, S. (1998) Effects of transcranial magnetic stimulation on severe depression. Similarities with ECT. Biological Psychiatry, 43, 765.

Hoffman, R. E., Boutros, N. N., Hu, S., et al (2000) Transcranial magnetic stimulation and auditory hallucinations in schizophrenia. Lancet, 355, 1073-1075

Klein, E., Kolsky, Y., Puyerovsky, M., et al (1999a) Right prefrontal slow repetitive transcranial magnetic stimulation in schizophrenia: a double-blind sham-controlled pilot study. Biological Psychiatry, 46, 1451-1454.

- Kreinin, I., Chistyakov, A., et al (1999b) Therapeutic efficacy of right prefrontal slow repetitive transcranial magnetic stimulation in major depression: a double-blind controlled study. Archives of General Psychiatry, 56, 315320 .

Lisanby, S. H. \& Sackeim, H. A. (2000) TMS in major depression. In: Transcranial Magnetic Stimulation in Neuropsychiatry (eds M. S. George \& R. H. Belmaker), pp. 185-200. Washington, DC: American Psychiatric Press.

- Luber, B., Perera, T., et al (2000) Transcranial magnetic stimulation: applications in basic neuroscience and neuropsychopharmacology. International Journal of Neuropsychopharmacology, 3, 259-273.

Loo, C., Mitchell, P., Sachdev, P., et al (1999) Double-blind controlled investigation of transcranial magnetic stimulation for the treatment of resistant major depression. American Journal of Psychiatry, 156, 946-948.

Lorberbaum, J. P. \& Wassermann, E. M. (2000) Safety concerns of TMS. In Transcranial Magnetic Stimulation in Neuropsychiatry (eds M. S. George \& R. H. Belmaker), pp. 141-162. Washington, DC: American Psychiatric Press.

Nahas, Z., Speer, A. M., Molloy, M., et al (1998) Preliminary results concerning the roles of frequency and intensity in the antidepressant effect of daily left prefrontal rTMS (abstract). Biological Psychiatry, 43, 315.

-, Bohning, D. E., Molloy, M. A., et al (1999) Safety and feasibility of repetitive transcranial magnetic stimulation in the treatment of anxious depression in pregnancy: a case report. Journal of Clinical Psychiatry, 60, 50-52.

-, Molloy, M., Risch, S. C., et al (2000) TMS in schizophrenia. In Transcranial Magnetic Stimulation in Neuropsychiatry (eds M. S. George \& R. H. Belmaker), pp. 237-252. Washington, DC: American Psychiatric Press.

Padberg, F., Zwanzger, P., Thoma, H., et al (1999) Repetitive transcranial magnetic stimulation (rTMS) in pharmacotherapy-refractory major depression: comparative study of fast, slow and sham rTMS. Psychiatry Research, 88, 163-171.

Pascual-Leone, A. \& Hallett, M. (1994) Induction of errors in a delayed response task by repetitive transcranial magnetic stimulation of the dorsolateral prefrontal cortex Neuroreport, 5, 2517-2520.

_- Houser, C., Reese, K., et al (1993) Safety of rapid-rate transcranial magnetic stimulation in normal volunteers. Electroencephalography and Clinical Neurophysiology, 89, 120-130.

_-, Rubio, B., Pallardó, F., et al (1996) Rapid-rate transcranial magnetic stimulation of left dorsolateral prefrontal cortex in drug-resistant depression. Lancet, 348, 233-237.

Paus, T. (1999) Imaging the brain before, during and after transcranial magnetic stimulation. Neuropsychologia, 37, 219-224.

Puri, B. K., Davey, N. J., Ellaway, P. H., et al (1996) An investigation of motor function in schizophrenia using transcranial magnetic stimulation of the motor cortex. British Journal of Psychiatry, 169, 690-695.

Rush, A. J., George, M. S., Sackeim, H. A., et al (2000) Vagus nerve stimulation (VNS) for treatment-resistant depressions: a multicenter study [see comments]. Biological Psychiatry, 47, 276-286.

Samii, A., Wassermann, E. M., Ikona, K., et al (1996) Decreased postexercise facilitation of motor evoked potentials in patients with chronic fatigue syndrome or depression. Neurology, 47, 1410-1414.

Shajahan, P. M., Glabus, M. F., Gooding, P. A., et al (1999a) Reduced cortical excitability in depression. Impaired postexercise motor facilitation with transcranial magnetic stimulation. British Journal of Psychiatry, 174, 449-454.

- - - Jenkins, J. A., et al (1999b) Postexercise motor evoked potentials in depressed patients, recovered depressed patients, and controls. Neurology, 53, 644-646.

Steele, J. D., Glabus, M. F., Shajahan, P. M., et al (2000) Increased cortical inhibition in depression: a prolonged silent period with transcranial magnetic stimulation (TMS). Psychological Medicine, 30, 565-570.

Ziemann, U. \& Hallet, M. (2000) Basic neurophysiological studies with TMS. In Transcranial Magnetic Stimulation in Neuropsychiatry (eds M. S. George \& R. H. Belmaker), pp. 45-98. Washington, DC: American Psychiatric Press.

\section{Multiple choice questions}

1. For the mechanism of action of TMS it is important to note that:

a a large supercooled magnet is necessary, as in MRI 
b the stimulation coil must touch the brain surface

c electromagnetic induction is involved

$\mathrm{d}$ the stimulation coil must not be electrically insulated

e loose dental fillings can be a problem.

2. Likely risks of TMS include:

a interference with cardiac pacemakers

$b$ induction of excitotoxicity

c memory loss

d complications of the general anaesthetic

e induction of seizures in predisposed patients.

3. In depression:

a TMS can be used to investigate brain mechanisms of the illness

$b$ there is overall no difference between active and sham treatments

c it is completely impossible for patients to distinguish sham and active treatment

$\mathrm{d}$ TMS is as effective as ECT in psychotic depression

e TMS's positive effects on mood are outweighed by its negative effects on memory.

4. TMS:

a is always likely to aggravate epilepsy

b can be used as a treatment of epilepsy

c has been used to treat positive and negative symptoms of schizophrenia d has no effects on mood in healthy volunteers

e has effects that vary with strength and frequency of the stimulation.

5. In a TMS investigation:

a central conduction time can be determined by stimulating over motor cortex and then over peripheral nerve roots

b cortical excitability can be determined using the effects of TMS on muscle activity

c everybody in the examination room has to wear industrial ear protectors

$\mathrm{d}$ pregnancy in the investigator or the patients is irrelevant

e only fully trained neurologists are able to conduct the examination.

\begin{tabular}{|llllllllll}
\hline MCQ answers & & & & & & & \\
1 & & 2 & & 3 & & 4 & & 5 & \\
a & F & a & T & a & T & a & F & a & T \\
b & F & b & F & b & F & b & T & b & T \\
c & T & c & F & c & F & c & T & c & F \\
d & F & d & F & d & F & d & F & d & F \\
e & F & e & T & e & F & e & T & e & F \\
\hline
\end{tabular}

